\title{
On the Generalized Arc Routing Problem
}

\author{
Elena Fernández \\ Department of Statistics and Operations Research \\ BarcelonaTech, Barcelona, Spain \\ Email: e.fernandez@upc.edu
}

\section{Introduction}

In this work we study the Generalized Arc Routing Problem (GARP) on an undirected graph. In the GARP a given graph is partitioned into clusters and a route is sought that traverses at least one edge of each cluster. Broadly speaking, the GARP is the arc routing counterpart of the Generalized Traveling Salesman Problem (GTSP). In the GTSP the set of vertices of a given graph is partitioned into clusters and a route is sought that visits at least one vertex of each cluster. Different versions of the GTSP have been studied by various authors (see, for instance, Noon and Bean, 1991; Laporte et al., 1987; Fischetti et al., 1995; Baldacci et al., 2010; Cacchiani et al., 2011).

Potential applications of the GARP arise in different contexts. In meter reading, one of the classical applications of arc routing, current technology allows to traverse just a few of the edges where meters are placed. Also in quality control for networks maintenance in which only a small subset of the edges of a network has to be traversed. Furthermore, the GARP is most appropriate for modeling location/arc-routing problems in which, due to their characteristics, facilities cannot be located at the nodes of a network, so they have to be located at the edges of some given areas (clusters) and a route connecting the facilities is sought. We introduce the GARP as a single vehicle arc routing problem on an undirected graph, and give a first linear integer formulation using two sets of binary variables, in the spirit of current formulations for this type of problems. After studying some optimality conditions, a tighter formulation with only one set of binary variables is proposed. The polyhedron associated with the latter formulation is studied and some facets and families of valid inequalities are given. In particular, we present two new families of inequalities which are valid for the GARP and extend the well-known co-circuit and matching inequalities, and we establish some relationship between them. We also study the separation problem for the different families of valid inequalities, and we propose a solution algorithm which iteratively reinforces the current LP relaxation by incorporating separated inequalities. Finally, we report on the numerical results of a series of computational experiments. 


\section{Problem definition}

The GARP is defined on an undirected connected graph $G=(V, E)$ with a distinguished vertex $v_{d} \in V$, the depot. With each edge $(u, v) \in E$ is associated a cost, $c_{u v} \geq 0$. A set of subgraphs of $G$ is given, $C_{k}=\left(V_{k}, D_{k}\right), k \in K$ with $V_{k} \subset V, \emptyset \neq D_{k} \subset E, k \in K$, and $V_{k} \cap V_{k^{\prime}}=\emptyset, k, k^{\prime} \in K, k \neq k^{\prime}$. Subgraphs $C_{k}=\left(V_{k}, D_{k}\right), k \in K$, are referred to as clusters and edges in $D=\cup_{k \in K} D_{k}$ as demand edges. We assume no demand edge is incident with the depot. If necessary, a new depot $v_{d}^{\prime}$ is defined and connected to the original one with a zero cost non-demand edge $\left(v_{d}, v_{d}^{\prime}\right)$ and with every other vertex $u \in V \backslash\left\{v_{d}\right\}$ with a non-demand edge $\left(u, v_{d}^{\prime}\right)$ of cost $c_{u v_{d}}$. We further assume that $G$ has been simplified so that $V$ is the set of vertices incident with edges in $D$ plus the depot, and $E$ contains the edges in $D$ plus additional non-demand edges, connecting every pair of vertices not connected by an edge of $D$, representing shortest paths in the original graph. This implies that the costs of non-demand edges satisfy the triangle inequality. Feasible solutions to the GARP are tours, passing through the depot, which traverse at least one edge of each cluster. The GARP is to find a minimum cost feasible tour. The GTSP on an undirected graph can be transformed into a GARP. Thus the GARP is NP-hard.

Similarly to other single vehicle arc routing problems on undirected graphs, for a given GARP instance, an optimal solution exists in which no edge is traversed more than twice. It is thus possible to formulate the GARP as a mixed integer program with two sets of variables representing the first and second traversal of edges. For $e \in E$, let $x_{e}$ and $y_{e}$ be indicator variables for the first and second traversal of edge $e$, respectively. For let $S \subset V$, $\delta(S)=\{e \in E \mid e=(i, j), i \in S, j \in V \backslash S\}$ denote the cut-set between $S$ and $V \backslash S$. Then, a formulation for the GARP is as follows:

$$
\begin{array}{rlrl}
\left(F_{x y}\right) \quad \min & \sum_{e \in E} c_{e}\left(x_{e}+y_{e}\right) & & \\
& x\left(D_{k}\right) \geq 1 \quad k \in K & & \\
& (x+y)(\delta(S)) \geq 2 & S=\bigcup_{k \in K_{S}} V_{k}, \quad K_{S} \subseteq K \\
& (x-y)(\delta(S) \backslash F)+y(F) \geq x(F)-|F|+1 & S \subset V, F \subseteq \delta(S),|F| \text { odd } \\
& y_{e} \leq x_{e}, & & \\
& x_{e}, y_{e} \in\{0,1\}, e \in E & &
\end{array}
$$

Inequalities (2) guarantee that at least one edge of each cluster is traversed, whereas connectivity with the depot is implied by constraints (3). Constraints (4) are an adaptation to the GARP of co-circuit inequalities (Barahona and Grötschel, 1986), which ensure even degree with respect to the solution of the visited vertices. These constraints, which

were proposed by Corberán et al. (2011b) for the Maximum Benefit Chinese Postman Problem, are a reinforcement of those used in Aráoz et al. (2009a,b) for the Clustered Prize- 
collecting Arc-routing Problem and the Privatized Rural Postman problem, respectively. They exploit the precedence relationship of the $x$ variables with respect to the $y$ variables, which is captured by inequalities (5). Formulation (1)-(6) involves $2|E|$ variables and a number of constraints of types (3) and (4) which is exponential on $|V|$.

\section{Dominance conditions and improved formulation}

Because of the triangle inequality assumption and because clusters are node-disjoint, an optimal GARP solution exists satisfying the following properties: (a) No consecutive demand edges are traversed; (b) Exactly one demand edge of each cluster is traversed; $(c)$ No consecutive non-demand edges are traversed; and $(d)$ No edge is traversed twice.

As a consequence, an optimal GARP tour exists which traverses exactly one demand edge of each cluster. In such an optimal tour, $(i)$ any visited vertex other than the depot is incident with exactly one demand and one non-demand edge, (ii) no non-demand edge connecting two vertices in the same cluster will be used, and (iii) any (demand or non-demand) edge is traversed at most once. By (ii) we can further simplify graph $G$ by removing any non-demand edge connecting two endnodes in the same cluster. This implies that the only non-demand edges of $E$ either connect vertices in different clusters or are incident with $v_{d}$. That is, $E$ contains the edges in $D$ plus additional non-demand edges representing shortest paths in the original graph, which connect the depot with any other vertex and every pair of vertices in different clusters. In the simplified graph we denote $n=|V|, m=|E|$, and $p=|K|$. Let also $R^{d}=\delta\left(v_{d}\right)$ and $R=E \backslash D$. For each $u \in V \backslash\left\{v_{d}\right\}$, $h(u)$ denotes the index of the cluster that contains $u$ (i.e. $u \in V_{h(u)}$ ). Taking into account the above properties we can build an improved formulation for the GARP which only uses one set $x$ of binary variables to indicate the edges that are traversed in the solutions. Let $P_{x y}$ denote the polyhedron associated with feasible solutions to formulation $F_{x y}$, and $P_{x}$ the polyhedron associated with the improved formulation which only uses binary variables associated with the edges that are traversed. The following properties hold:

1. The feasible domain for $P_{x}$ is contained in the projection of the feasible domain for $P_{x y}$ onto the subspace $\left\{y_{e}=0, e \in E\right\}$. That is: $P_{x} \subseteq P_{x y} \cap\left\{y_{e}=0, e \in E\right\}$. The same applies to their linear programming (LP) relaxations.

2. $\operatorname{dim}(P)=m-p-(n-1)$.

3. The following families of inequalities are facets of $P_{x}:(i)$ Non-negativity inequalities $x_{e} \geq 0, e \in E$; and $(i i)$ Connectivity inequalities $x(\delta(S)) \geq 2$, with $S=$ $\bigcup_{k \in K_{S}} V_{k}, K_{S} \subseteq K,|K| \geq 2$.

4. The following families of inequalities are valid for $P_{x}$ : 
(a) $x(\delta(S)) \geq 2 x_{e} \quad S \subseteq V \backslash\left\{v_{d}\right\}, e \in E(S)$.

(b) Parity inequalities: $x(\delta(S) \backslash F) \geq 1-|F|+x(F) \quad S \subset V, F \subseteq \delta(S),|F|$ odd.

(c) Matching inequalities: $x(E(S) \cap R) \leq \frac{r-1}{2}$, for $S=\left\{v_{1}, \ldots v_{r}\right\} \subset V \backslash\left\{v_{d}\right\}$, with $r$ odd and $h\left(v_{i}\right) \neq h\left(v_{j}\right)$, for $i \neq j$.

5. By taking into account the optimality conditions $(a)-(d)$, parity inequalities and matching inequalities can be reinforced to stronger inequalities. In some cases, a relation between both types of reinforced inequalities can be established.

\section{References}

J. Aráoz, E. Fernández and C. Franquesa. The Clustered Prize-collecting Arc-routing Problem, Transportation Science 43, 287-300, 2009a.

J. Aráoz, E. Fernández, O. Meza. An LP-based algorithm for the privatized rural postman problem. European Journal of Operational Research 196(3) 886-896, 2009b.

R. Baldacci, E. Bartolini and G. Laporte. Some applications of the generalized vehicle routing problem. Journal of the Operational Research Society 61, 1072-1077 (2010).

F. Barahona and M. Grötschel. On the cycle polytope of a binary matroid. J. Comb. Theory 40 40-62, 1986.

V. Cacchiani, A.E. Fernandes Muritiba, M. Negreiros, P. Toth. A multistart heuristic for the equality generalized traveling salesman problem. Networks 57, 231-239, 2011.

Á. Corberán, E. Fernández, C. Franquesa, and J.M. Sanchis. The windy clustered prizecollecting arc routing problem. Trans. Sci. 45, 317-344, 2011a.

Á. Corberán, I. Plana, A. Rodríguez-Chía, J.M. Sanchis. A Branch-and-Cut for the Maximum Benefit Chinese Postman Problem. Math. Program., DOI: 10.1007/s10107-0110507-6, 2011b.

M. Fischetti, J.J. Salazar-González, and P. Toth. The symmetric generalized traveling salesman polytope. Networks 26 113-123, 1995.

G. Ghiani and G. Laporte. A branch-and-cut algorithm for the undirected rural postman problem. Math. Program. 87, 467-481, 2000.

G. Laporte, H. Mercure, and Y. Nobert. Generalized traveling salesman problem through n sets of nodes: The asymmetrical case. Discrete Appl Math 18 185-197, 1987.

C.E. Noon and J.C. Bean. A Lagrangian based approach for the asymmetric generalized traveling salesman problem. Operations Research 39 623-632, 1991. 